\title{
ANÁLISE DA PRECIPITAÇÃo NA CIDADE DE SÃO PAULO NO PERÍODO 1961-2009
}

\author{
Mozar de Araújo Salvador ${ }^{1}$ \\ Larissa Sayuri Futino Castro dos Santos ${ }^{2}$
}

\begin{abstract}
RESUMO
O objetivo do estudo foi verificar possíveis mudanças no comportamento da precipitação pluviométrica na Cidade de São Paulo através de índices climáticos de extremos propostos pelo ETCCDI (Expert Team on Climate Change Detection and Indices) e aplicados a uma série de dados diárioS da estação meteorológica 83781 durante o período 1961-2009 utilizando a ferramenta RClindex. Foi observado que não houve qualquer tendência significativa no número de dias úmidos, porém o volume total anual de precipitação apresentou uma tendência significativa de aumento. Isso teve como causa o aumento no número de dias com chuvas intensas detectadas pelos índices R20mm, R50mm e R95p.
\end{abstract}

Palavras-chave: Clima, precipitação e RClindex.

\begin{abstract}
The aim of this study was to evaluate possible changes in the precipitation behavior in São Paulo city using climate extremes indices proposed by ETCCDI (Expert Team on Climate Change Detection and Indices) and applied to a data set of daily weather station 83781 during the period 1961-2009 using the tool RClindex. It was observed that there was no significant trend in the number of wet days, but the total annual volume of precipitation showed a significant trend of increase. This was due to increase in the number of days with heavy rainfall detected by R20mm, R50mm and R95p.
\end{abstract}

Key words: Climate, precipitation and Rclindex.

\footnotetext{
1 Mestre em Meteorologia - Meteorologista-Instituto Nacional de Meteorologia-INMET, mozar.salvador@inmet.gov.br.

2 Graduanda em Estatística - Universidade de Brasília e estagiária do INMET, larissa.santos@inmet.gov.br.
} 


\section{INTRODUÇÃO}

A discussão acerca das mudanças ocorridas no clima global tem dominado o debate científico da última década. Não obstante, pesquisas sobre o comportamento climático em escalas menores (regional ou local) têm crescido motivadas pela necessidade de se detectar e compreender os aspectos pontuais de tais mudanças em áreas de grande importância, seja pelo seu aspecto ambiental, como nas florestas tropicais ou pelo aspecto econômico-social, como nas metrópoles e nas grandes áreas de produção agrícola. Nesse contexto, a cidade de são Paulo, por sua importância sócio-econômica, passa a ser objeto de pesquisas nas mais diversas áreas de estudo, incluindo a Climatologia, que buscam compreender os possíveis reflexos do seu desenvolvimento no meio ambiente.

No Brasil, vários estudos recentes foram feitos visando identificar alterações ou variabilidades climáticas em diversas localidades. Estudo apresentado por Siqueira et al (2006) demonstrou, através de uma análise de séries de dados de 40 anos, que a precipitação em parte das regiões Norte e Nordeste está sob a influência da Oscilação Decadal do Pacífico (MANTUA et al., 1997). Santos e Brito (2007) também detectaram aumento da precipitação anual nos estados da Paraíba e Rio Grande do Norte correlacionado com as anomalias de temperatura da superfície do mar. Dufek e Ambrizzi (2008) observaram que o volume anual de chuvas e as precipitações intensas apresentaram um crescimento no Estado de São Paulo no período de 1950 a 1999. Santos et al. (2009) analisaram a tendência dos índices de extremos no Estado do Ceará e detectaram aumento de precipitação em alguns pontos do estado.

Uma das dificuldades encontradas nas pesquisas climáticas é a de se reunir um conjunto consistente de dados de longo período sem ou com poucas falhas. Dados de natureza temporal, como os dados meteorológicos, são constantemente comprometidos pelo problema de dados faltantes, uma vez que a não observação de determinada variável pode ser conseqüência da falta do observador, de uma medição incorreta por parte do instrumento de medição ou mesmo do seu não funcionamento.

Nas últimas décadas grande esforço foi empregado na estatística intuindo desenvolver métodos que lidem com essa dificuldade. Uma abordagem comprovadamente apropriada é imprescindível uma vez que há a possibilidade de se tomar conclusões equivocadas caso o método empregado seja inadequado.

A falta de uma dada observação, ou ainda de várias, é comumente contornada utilizando-se como base para o estudo apenas o banco completo, ou seja, apenas os dados observados. Entretanto, tal análise é incorreta já que a realização da série temporal em estudo é considerada uma amostra dentre todas as possíveis realizações da série daquela variável e a perda de informações aumenta proporcionalmente a redução da amostra, diminuindo, conseguintemente, o poder estatístico do estudo, o que justificaria a utilização de métodos de imputação (NUNES et al., 2001). Outra justificativa bastante pertinente para optar pela imputação dos dados nesse estudo é que o software utilizado, Rclimdex, tem limitações quanto ao número de observações faltantes.

Deve-se salientar que a análises de um banco de dados completo (considerando apenas os dados efetivamente observados) e a de um banco de 
dados imputado diferem na maioria dos casos e que o pesquisador deve considerar as diferenças entre as duas análises.

O objetivo do estudo foi verificar possíveis mudanças nas características e no comportamento da precipitação pluviométrica da maior cidade do Brasil através de índices climáticos de extremos propostos por pesquisadores canadenses do ETCCDI (Expert Team on Climate Change Detection and Indices) e aplicados a série de dados diário da estação meteorologia 83781 na Cidade de São Paulo.

\section{DADOS E MÉTODOS}

Os dados diários de precipitação pluviométrica utilizados são da estação meteorológica 83781-São Paulo (Mirante de Santana), no período 1961-2009. Os períodos com dados faltantes foram preenchidos com o método imputação simples de estações mais próximas, do Departamento de Água e Energia Elétrica do Estado de São Paulo (DAEE) e de reanálise em ponto de grade do Climate Prediction Center do Nacional Oceanic and Atmospheric Administration (CPC/NOAA). As informações sobre cada estação meteorologia utilizadas, bem como sobre os dados de ponto de grade do CPC, encontram-se na Tabela 1.

Tabela 01 - Identificação das séries de dados utilizados, suas coordenadas, período e instituição pertencente.

\begin{tabular}{|c|c|c|c|c|}
\hline Estação & Latitude & Longitude & Período & Instituição \\
\hline $\begin{array}{l}\text { 83781-São Paulo (Mirante } \\
\text { Santana) }\end{array}$ & $23^{\circ} 30^{\prime}$ & $46^{\circ} 37^{\prime}$ & 1961-2009 & INMET \\
\hline E3-050-Arujá & $23^{\circ} 23^{\prime}$ & $46^{\circ} 21^{\prime}$ & 1969-1992 & DAEE \\
\hline E3-047- Franco da Rocha & $23^{\circ} 20^{\prime}$ & $46^{\circ} 41^{\prime}$ & 1961-1992 & DAEE \\
\hline E3-091- Itaquaquecetuba & $23^{\circ} 29^{\prime}$ & $46^{\circ} 22^{\prime}$ & 1961-1992 & DAEE \\
\hline $\begin{array}{l}\text { Ponto de grade mais } \\
\text { próximo }\end{array}$ & $23^{\circ} 30^{\prime}$ & $46^{\circ} 30^{\prime}$ & 1961-1999 & CPC/NOAA \\
\hline
\end{tabular}

\section{a) Imputação}

Como previamente citado, diversas são as técnicas disponíveis para imputação de modo que ao se optar por trabalhar com dados imputados há a necessidade de estudar ou mesmo escolher o método a ser empregado. Há a possibilidade de efetuar tal escolha baseando-se exclusivamente na proporção de dados faltantes. Segundo Harrell Jr. (2001):

i. Se a proporção for menor ou igual a 0,05: Pode-se utilizar o método de imputação única ou ainda considerar apenas o banco de dados completo;

ii. Se a proporção estiver entre 0,05 e 0,15: É possível utilizar o método de imputação única sendo aconselhável o método de imputação múltipla e

iii. Se a proporção for maior ou igual a 0,15: Indica-se o uso de imputação múltipla.

Na série temporal apresentada, o número de dias cuja variável não foi observada se aproxima de 365, em uma série com 17897 dias (49 anos), compreendendo medições de 1961 a 2009. Logo, a proporção de dados não observados é de $2,04 \%$ do total. Na regra anteriormente citada, seria possível 
trabalhar com os dados exclusivamente observados ou preenche-los pelo método de imputação única. A primeira opção foi desconsiderada pelos motivos apresentados nesse documento.

A denominada imputação única corresponde à técnica mais simples de imputação. Nela, os dados faltantes são substituídos pela média ou mediana ou mesmo uma regressão linear dos dados observados. A imputação é realizada apenas uma vez e utiliza-se o banco completo para dar andamento ao estudo. Ressalva-se a sua simplicidade e a importância de empregá-la exclusivamente para a pequena proporção de dados faltantes (NUNES et al., 2001).

Empregar tal método sugeriu a escolha de algumas estações próximas à estação de interesse para que elas servissem como doadoras de informações. 0 intuito é observá-las em um dia no qual não há observação da série original e substituir pela média das observações das estações doadoras. Uma escolha adequada dessas estações é imprescindível, já que se deve preservar uma semelhança entre as suas séries. Há similaridade, sobretudo, entre séries próximas geograficamente sendo conveniente, portanto, escolher aquelas mais próximas possíveis para que se obtenha informação de uma mesma região climática homogênea. No caso em estudo foram escolhidas séries de estações convencionais vizinhas do DAEE e um ponto grade dos dados de reanálise CPC sobre a cidade de São Paulo. Para cada dia faltante a forma como foi realizada a imputação pode ser descrito como:

i. Se três ou duas das estações convencionais selecionadas têm observação referente ao dia faltante fez-se uma média aritmética entre essas observações para substituir a observação faltante. Esta foi a ação predominante no preenchimento de falhas.

ii. Se apenas uma das estações convencionais tinha a observação do dia faltante em questão, utilizou-se também o dado do ponto gradeado. Procedeu-se com a média entre esses dois valores para substituir na série original.

iii. Se nenhuma estação convencional vizinha possuía a observação do dia faltante na série a ser imputada utilizou-se exclusivamente o ponto gradeado como doador. O seu valor foi substituído na série sem sofrer qualquer alteração.

Uma vez que o valor não observado foi substituído e completou-se a série temporal, as análises foram realizadas normalmente, como prevê o método de imputação única.

\section{b) Índices climáticos}

Para se obter uma perspectiva uniforme sobre as variações e extremos climáticos, o ETCCDI (Expert Team on Climate Change Detection and Indices) definiu um conjunto de índices indicadores descritivos de extremos. Tais índices descrevem as características particulares de extremos, incluindo a freqüência, amplitude e persistência. O conjunto inclui 27 índices de extremos, sendo 16 para temperatura e 11 para precipitação. As especificações para todos esses índices podem ser obtidas a partir do sítio http://cccma.seos.uvic.ca/ETCCDI.

Neste trabalho, a análise dos dados diários foi feita com base em alguns dos índices de precipitação propostos pelo ETCCDI e calculados através do software RClindex 2.3.1 (ZHANG e YANG, 2004). Os índices utilizados para este 
estudo foram: 1) PRCPTOT - precipitação total anual; 2) R1 mm - número de dias com precipitação significativa igual ou maior que $1 \mathrm{~mm}$; 3) R10 $\mathrm{mm}$ número de dias com precipitação igual ou maior que $10 \mathrm{~mm}$, 4) R20 mm número de dias com precipitação igual ou maior que $20 \mathrm{~mm}, 5)$ R50 mm número de dias com precipitação igual ou maior que $50 \mathrm{~mm}, 6$ ) SDII - índice simples de intensidade diária e 7) R95p - precipitação total de dias muito úmidos acima do percentil 95. A descrição detalhada é apresentada na Tabela 2.

Tabela 2 - Descrição dos índices climáticos utilizados

\begin{tabular}{|c|c|c|c|}
\hline Índice & Significado do índice & Definição & Unidade \\
\hline PRCPTOT & $\begin{array}{c}\text { Precipitação total anual dos dias } \\
\text { úmidos. }\end{array}$ & $\begin{array}{l}\text { Precipitação anual total de dias úmidos } \\
\text { (precipitação }>=1 \mathrm{~mm} \text { ) }\end{array}$ & $\mathrm{Mm}$ \\
\hline R1mm & $\begin{array}{l}\text { Número de dias úmidos com } \\
\text { precipitação }>\text { ou }=1 \mathrm{~mm} \text {. }\end{array}$ & $\begin{array}{l}\text { Número de dias em um ano em que } \\
\text { Precipitação> =1 mm. }\end{array}$ & Dias \\
\hline $\mathrm{R} 10 \mathrm{~mm}$ & $\begin{array}{l}\text { Número de dias com precipitação } \\
>\text { ou }=1 \mathrm{~mm} .\end{array}$ & $\begin{array}{l}\text { Número de dias em um ano em que } \\
\text { Precipitação }>=10 \mathrm{~mm} \text {. }\end{array}$ & Dias \\
\hline R20mm & $\begin{array}{l}\text { Número de dias com precipitação } \\
\qquad>\text { ou }=1 \mathrm{~mm} .\end{array}$ & $\begin{array}{l}\text { Número de dias em um ano em que } \\
\text { Precipitação }>=20 \mathrm{~mm} \text {. }\end{array}$ & Dias \\
\hline $\mathrm{R} 50 \mathrm{~mm}$ & $\begin{array}{l}\text { Número de dias com precipitação } \\
>>\text { ou }=1 \mathrm{~mm} .\end{array}$ & $\begin{array}{l}\text { Número de dias em um ano em que } \\
\text { Precipitação }>=50 \mathrm{~mm} .\end{array}$ & Dias \\
\hline SDII & $\begin{array}{c}\text { Índice simples de intensidade } \\
\text { diária }\end{array}$ & $\begin{array}{l}\text { Precipitação anual total dividida pelo o } \\
\text { número de dias úmidos (definidos por } \\
\text { PRCP }>=1.0 \mathrm{~mm} \text { ) em um ano. }\end{array}$ & mm.dia ${ }^{1}$ \\
\hline R95p & Dias muito úmidos. & $\begin{array}{l}\text { Precipitação anual total em que a } \\
\text { precipitação é >95 percentil. }\end{array}$ & $\mathrm{Mm}$ \\
\hline
\end{tabular}

Como acréscimo, também foi feita uma análise comparativa entre as médias das PRCPTOT de quatro períodos de 10 anos (1961-1970, 1971-1980, 1981-1990 e 1991-2000) e um período de 9 anos (2001-2009) com a média de total da série, visando, tão-somente, detectar as variações climáticas existentes entre períodos longos dentro da série utilizada.

\section{c) Teste de hipótese}

Usualmente em uma pesquisa há a suspeita sobre um determinado comportamento de uma ou várias variáveis (BUSSAB e MORETTIN, 2006). A análise de tal suspeita baseia-se na amostra de observações retiradas da população. O intuito principal de um teste estatístico de hipótese é fornecer uma metodologia que permita verificar se os resultados dessa amostra vão ao encontro ou não de tal suspeita. Grosso modo, o teste supõe verdadeira a hipótese (suspeita) que se tem e constata se a porção retirada é plausível sobre ela. O pesquisador descreve a negação do que se deseja provar em termos da hipótese nula $\left(\mathrm{H}_{0}\right)$, ou seja, a hipótese que verdadeiramente se deseja testar é a hipótese alternativa $\left(H_{1}\right)$. Há, contudo, a chance de que se rejeite $H_{0}$ para um determinado nível de significância, mas que não o rejeite para outro menor. Tal dificuldade pode ser sanada valendo-se do $p$-valor ou probabilidade de significância. Essa medida é a probabilidade de que o teste acuse um resultado mais desfavorável possível a $\mathrm{H}_{0}$ do que o resultado apresentado pela amostra tirada. Ou ainda, segundo Barbetta (2006), poder-se-ia dizer que p-valor é "o risco de se tomar a decisão errada após a observação da amostra, caso se rejeite $\mathrm{H}_{0}$ ". Quanto menor for $\mathrm{p}$-valor, maior a evidência para se rejeitar a hipótese nula. 
Frequentemente, em estudos climáticos, fixa-se o nível de significância do teste (probabilidade de se rejeitar $\mathrm{H}_{0}$ dado que ele é verdadeiro) em 0,05 ou 0,10 . Sendo considerado, neste estudo, como não significativo qualquer valor acima 0,05 .

Especificamente no trabalho apresentado, fizeram-se testes de hipóteses para o coeficiente angular das funções de regressão estimadas (de tendência). Ou mais precisamente, traduzimos a possibilidade de se representar a série a partir de um função linear $\left(\beta_{1} x+\beta_{0}\right.$, onde $\beta_{1}$ é o coeficiente angular) pelas hipóteses:

$\mathrm{H}_{0}: \beta_{1}=0 \mathrm{e}$

$H_{1}: \beta_{1} \neq 0$, sendo $\beta_{1}$ o coeficiente angular.

Diz-se, ainda, que se testa se o modelo reduzido, aquele sob $\mathrm{H}_{0}$, é plausível. Caso o seja, pode-se afirmar que os dados não estão dispersos ao redor de uma reta de tendência, mas comportam-se aleatoriamente ao redor de uma constante.

\section{RESULTADOS E DISCUSSÃO}

A Tabela 2 apresenta o tipo de tendência (positiva ou negativa) e o pvalor de cada índice calculado. Também apresenta se a tendência foi significante ou não, considerando o nível de significância de 5\%. Os resultados apresentam, de forma geral uma tendência positiva significante ( $\leq \leq 0,05)$, excetuando-se 0 índice $\mathrm{R} 1 \mathrm{~mm}$.

Tabela 3 - P-valor e coeficiente angular de cada índice avaliado.

\begin{tabular}{ccc}
\hline Índice & P-valor & $\begin{array}{c}\text { Coeficiente angular } \\
\left(\boldsymbol{\beta}_{\mathbf{1}}\right)\end{array}$ \\
\hline PRCPTOT & 0,002 & 8,271 \\
\hline R1mm & 0,267 & 0,142 \\
\hline R10mm & 0,011 & 0,207 \\
\hline R20mm & 0,003 & 0,174 \\
\hline R50mm & 0,021 & 0,051 \\
\hline R95p & 0,006 & 4,231 \\
\hline SDII & 0,0 & 0,060 \\
\hline
\end{tabular}

O volume total anual de chuvas significativas (PRCPTOT) na cidade de São Paulo apresentou, até 2009, um comportamento de crescimento ao longo de toda a série estuda (Figura 01). Tal comportamento é inicialmente demonstrado pela reta de tendência linear acentuada e $p$-valor associado, foi de apenas 0,002 (Tabela 02). Isso indica, de uma forma geral, que se pode rejeitar a hipótese nula - hipótese de não haver inclinação na reta de tendência linear. 


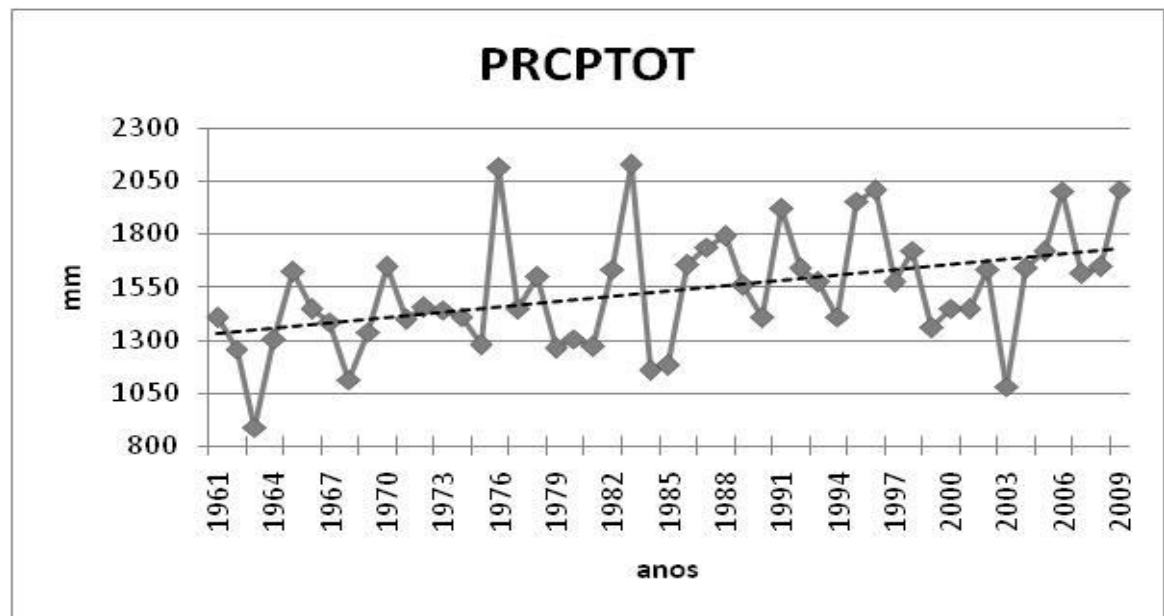

Figura 01. Precipitação total anual de dias úmidos (precipitação >=1 mm).

Nos 10 primeiros anos da série (1961-1970) a média foi de $1340 \mathrm{~mm}$, e nos últimos 10anos (1999-2009) a média foi de $1558 \mathrm{~mm}$. A média do índice PRCPTOT de para todo o período (1961-2009) foi de $1520 \mathrm{~mm}$. A Figura mostra que o índice PRCPTOT médio ficou abaixo de $1500 \mathrm{~mm}$ nos dois primeiros grupos de 10 anos e nos dois últimos, a média superou o volume de $1600 \mathrm{~mm}$.

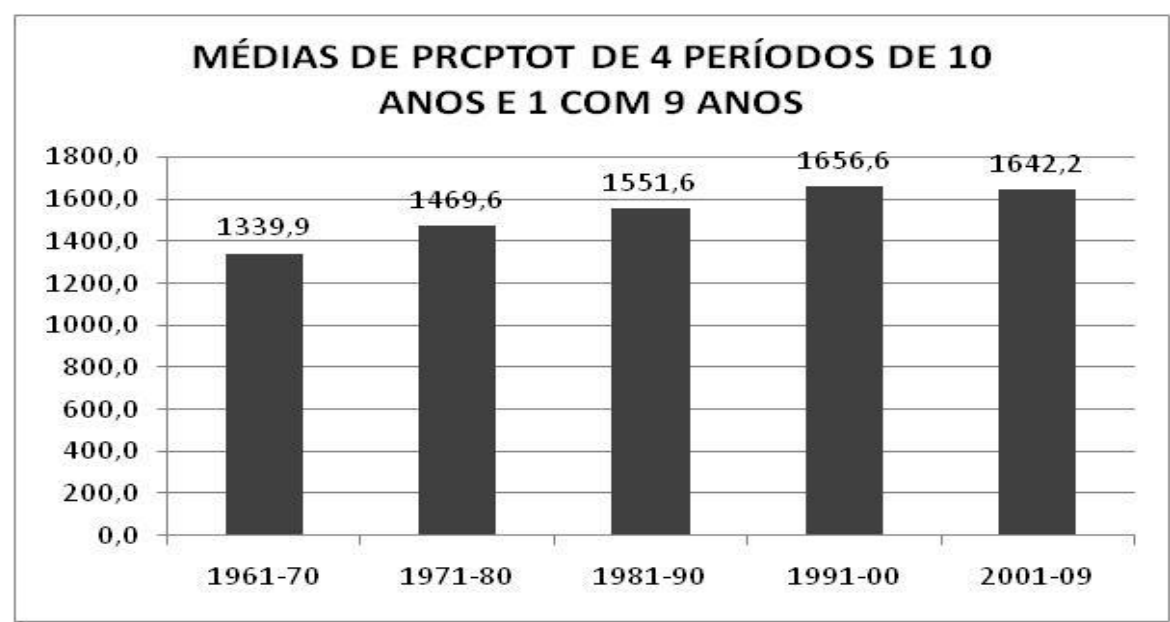

Figura 02. Média de por grupos de 10 e 9 anos do índice PRCPTOT.

Diferentemente, o gráfico do índice $\mathrm{R} 1 \mathrm{~mm}$, que indica a quantidade de dias úmidos (precipitação $\geq 1 \mathrm{~mm}$.Dia- ${ }^{1}$ ), não apresentou qualquer tendência significativa e, apesar de ter um coeficiente angular positivo, o alto $p$-valor associado $(0,267)$ permite afirmar que tal resultado é meramente conseqüência da aleatoriedade da série, e não de um comportamento constante. 


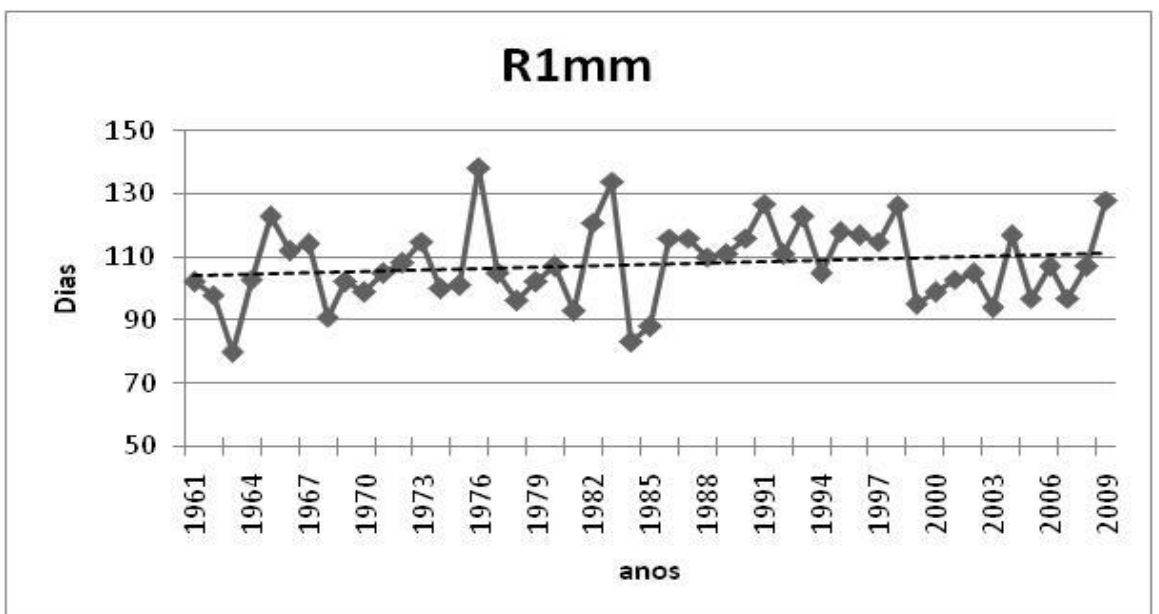

Figura 03. Gráfico de ocorrência de chuvas diárias iguais ou superiores a 1 $\mathrm{mm}$.

Descritivamente, observa-se que os anos de 1963 e 1984 foram os que apresentaram menos dias com precipitação significativa no ano. Na contramão, os anos de 1976 e 1983 foram os que apresentaram mais dias com precipitação igual ou superior a $1 \mathrm{~mm}$ ou, simplesmente, os anos com mais dias com chuvas significativas. Atentando-se para a função de regressão estimada apresentada, não é possível observar explicitamente um comportamento de crescimento ou declínio da variável. Tal fato é confirmado pela estimativa do coeficiente angular apresentado de 0,142 e pelo p-valor associado de 0,267, bem acima de 0,05. A partir dessa probabilidade de significância pode-se afirmar que não se rejeita a hipótese de que o coeficiente angular dessa função linear seja nulo $\left(\mathrm{H}_{0}=0\right)$. Desse modo, há evidências estatísticas de que os dados não possam ser representados por uma função de regressão linear estimada e que o comportamento da variável seja meramente aleatório.

Há, portanto, claras evidências de que a precipitação acumulada em um ano aumentou no decorrer da série, entretanto, o número de dias nos quais ocorreu precipitação significativa permaneceu inalterado. Dessa forma, é natural supor que a tendência de crescimento dos volumes anuais não se deu dependente do número de dias úmidos, mas em decorrência da intensificação da ocorrência de um maior volume de chuvas em um dia, como pode ser verificado nos índices $\mathrm{R} 20 \mathrm{~mm}$ e R50mm.

Referentemente ao número de dias por ano em que precipitação é igual ou superior a $10 \mathrm{~mm}$, o índice $\mathrm{R} 10 \mathrm{~mm}$, pode-se avaliar a pertinência de tal suspeita. O ano no qual ocorreram menos dias com precipitação maior ou igual a $10 \mathrm{~mm}$ foi 1963 e os anos com mais dias foram 1976 e 1983 . A mera análise gráfica de tal variável já levaria a concluir que a série apresentada se comporta de forma crescente. Coerentemente, o coeficiente de inclinação de 0,207 e o pvalor associado é de 0,011. Para esse valor, é possível afirmar que o modelo reduzido tem significância e que há evidências estatísticas de que os dados não estejam aleatoriamente dispersos ao redor de uma tendência linear. O número de dias com ocorrência de chuva de $10 \mathrm{~mm}$ ou mais sofreu alteração no decorrer da série de modo que a variável apresentou crescimento no período de 1961 a 2009. 


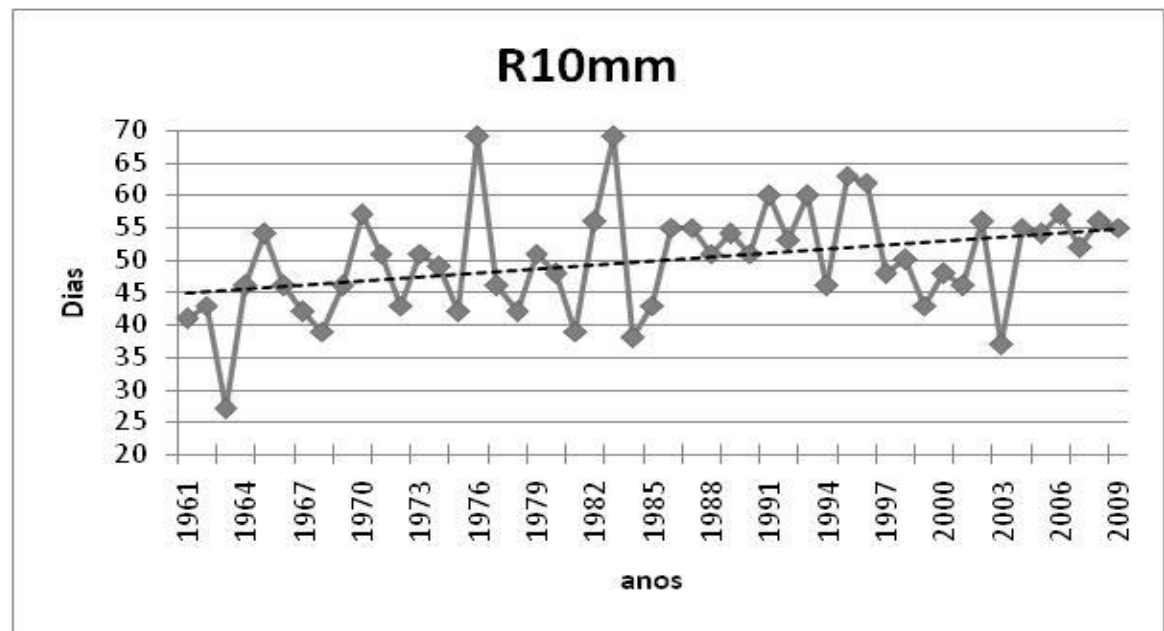

Figura 04. Gráfico de ocorrência de chuvas diárias iguais ou superiores a 10 $\mathrm{mm}$.

Quanto ao índice de número de dias por ano em que a precipitação é igual ou superior a $20 \mathrm{~mm}$, observa-se, igualmente, que os anos com menos ocorrências de tal variável foram 1963, com 13, seguido de 1968, com 15, e os anos com maiores ocorrências foram 1996, com 39, 1983 e 1995, ambos com 38 e 1996, com 35. Ao se visualizar a representação gráfica (Figura 05) tem-se a evidência de que a série tem modificado seu comportamento ao longo dos anos. O coeficiente angular positivo da reta foi de 0,174 , e a da probabilidade de significância associado, p-valor, de apenas 0,003. O baixíssimo p-valor encontrado leva à rejeição da hipótese de que não há tendência linear na série. Dessa forma, há evidências estatísticas que permitem afirmar que esse tipo de precipitação vem aumentando no decorrer da série. Portanto, o número de dias em um ano com chuvas iguais ou superiores a $20 \mathrm{~mm}$ apresenta-se de maneira crescente na série de 1961 a 2009 na cidade de São Paulo.

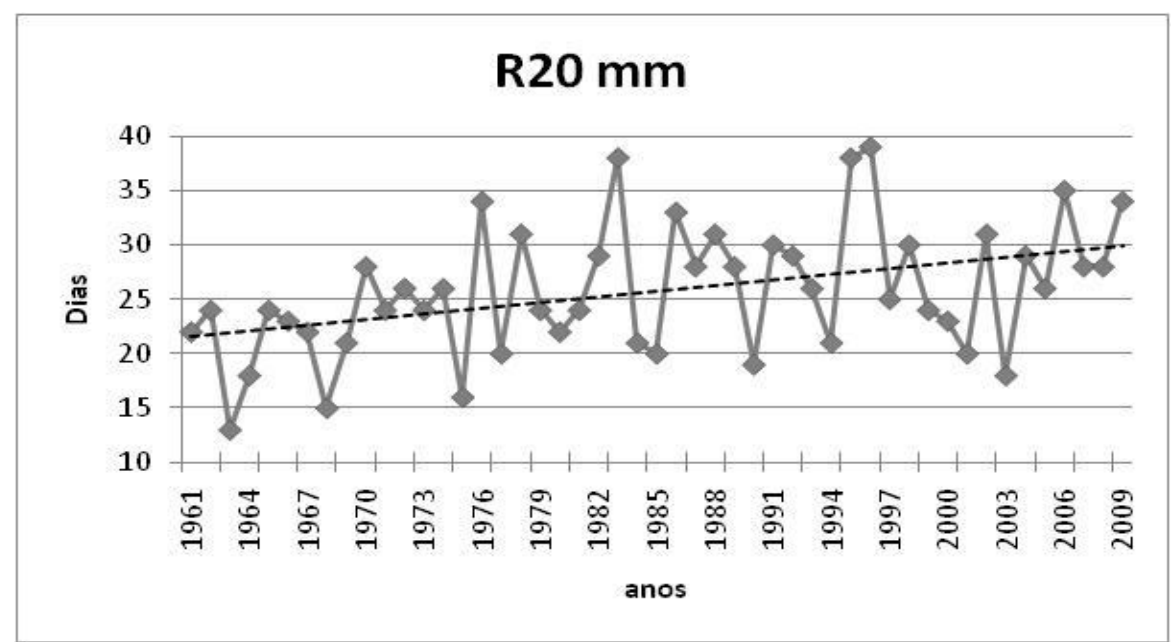

Figura 05. Gráfico de ocorrência de chuvas diárias iguais ou superiores a 20 $\mathrm{mm}$. 
O índice R50mm, número de dias por ano em que precipitação é igual ou superior a $50 \mathrm{~mm}$, reforça a hipótese da relação entre as chuvas mais intensas e o volume total anual. O seu gráfico (Figura 06) revela que os anos nos quais ocorreram mais vezes tal precipitação foram 1978, com 10, e 1996, com 11, seguidos dos anos 2006 e 2009, ambos com 8 ocorrências. Observa-se no gráfico que, até 1977, as ocorrências não ultrapassavam o número de 5 , enquanto nos últimos 5 anos foram de no mínimo 4 ocorrências por ano.

A estimativa do coeficiente angular dessa reta é de 0,051 e a probabilidade de significância associada à estimativa desse parâmetro é de 0,021 . Dessa maneira, rejeitou-se a hipótese de que não há uma tendência na série, demonstrando que as chuvas intensas, com $50 \mathrm{~mm}$ ou mais por dia, apresentou evidências estatísticas crescimento no período 1961-2009.

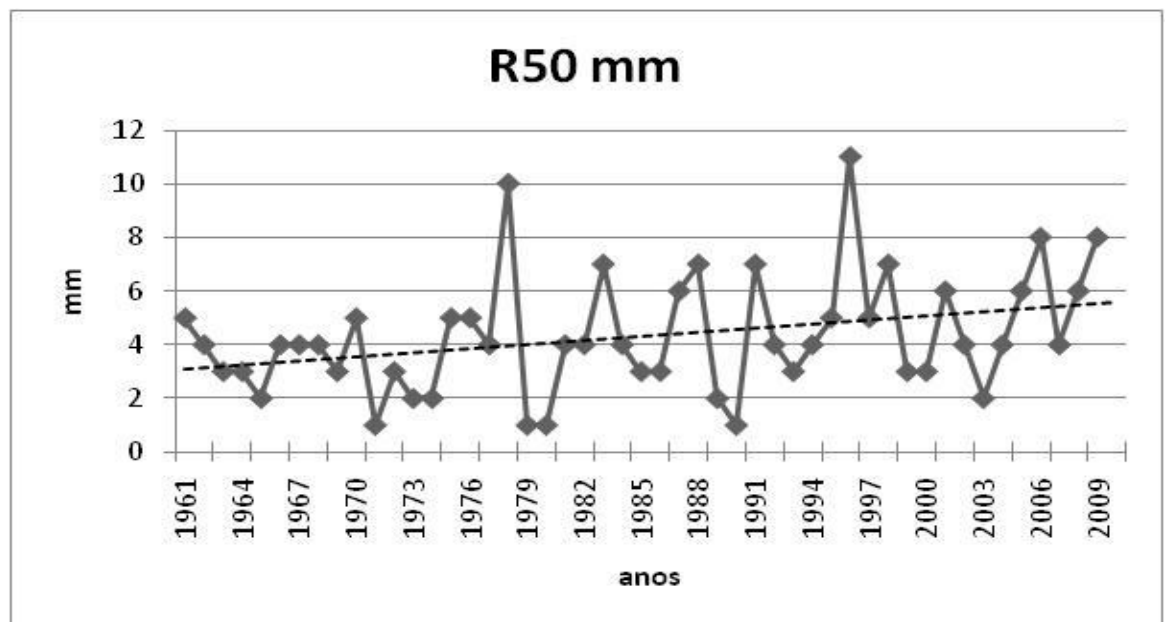

Figura 06. Gráfico de ocorrência de chuvas diárias iguais ou superiores a 50 $\mathrm{mm}$.

Outro índice verificado para auxiliar na compreensão da série foi o TR95p, precipitação anual total em que a precipitação é >95 percentil. A série desse índice reflete se as chuvas mais intensas, as $5 \%$ mais fortes, estão aumentando seu volume acumulado no decorrer do período apresentado. $O$ gráfico dá indícios de que tal suspeita esteja correta e a estimativa do coeficiente angular da reta, de 4,231 e seu p-valor associado, de apenas 0,006, também o fazem. Pelo $\mathrm{p}$-valor apresentado pode-se afirmar que há indícios estatísticos de que o comportamento da variável apresentou tendência de mudança e que essa, efetivamente, não foi mero resultado do comportamento aleatório da variável. Deve-se atentar para os anos de 1980 e 2003 nos quais o acumulado dos $5 \%$ de chuvas mais intensas foi bastante inferior ao acumulado da série em geral. Os três anos cujo acumulado das chuvas mais intensas foi maior foram 2006, 1996 e 2005, respectivamente $673,1 \mathrm{~mm}, 664,8 \mathrm{~mm}$ e $835,8 \mathrm{~mm}$.

Por fim, foi analisado o índice SDII (intensidade diária por ano), precipitação anual total dividida pelo o número de dias úmidos (precipitação $>=1.0 \mathrm{~mm}$ ) em um ano. Trata-se de uma de uma verificação simples da intensidade das chuvas significativas diárias (dias úmidos) medida em $\mathrm{mm}$.dia ${ }^{-1}$. A reta de tendência apresentou um coeficiente angular de 0,06 e 
o p-valor associado de 0 (zero). Dessa forma, rejeitou-se claramente a hipótese de que não há uma tendência significativa na série, demonstrando a existência de um comportamento crescente do indicie de intensidade diária de precipitação. As duas maiores intensidade foram em 2005 e 2006, respectivamente 17,7 e $18,7 \mathrm{~mm}^{\mathrm{d}} \mathrm{dia}^{-1}$.

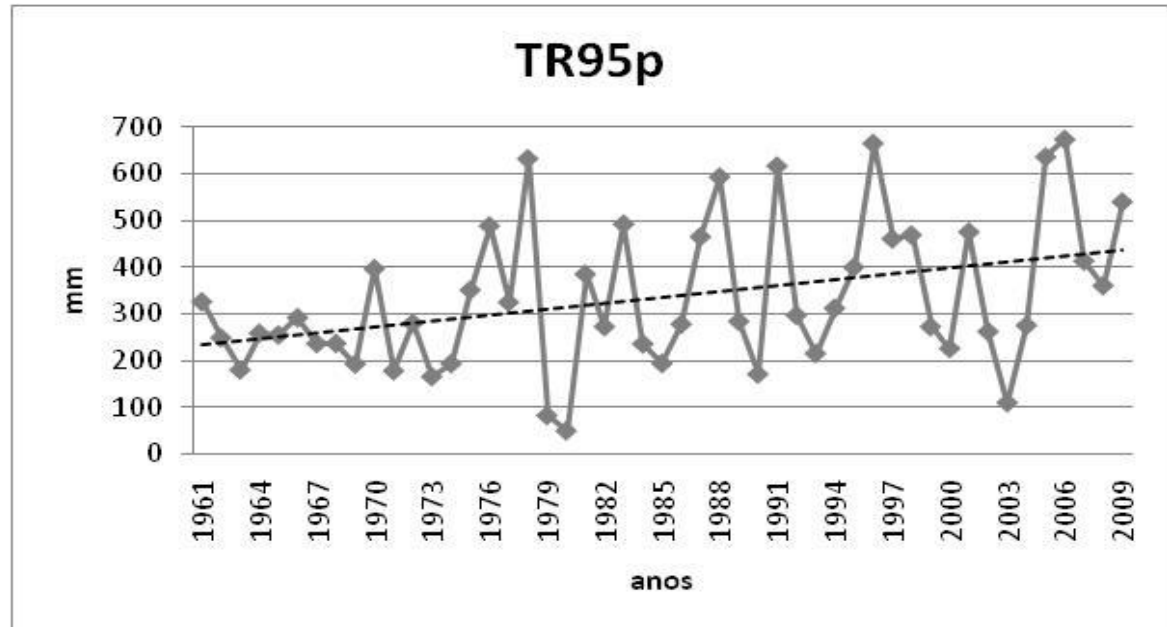

Figura 07. Gráfico do total da precipitação anual em que a precipitação é >95 percentil.

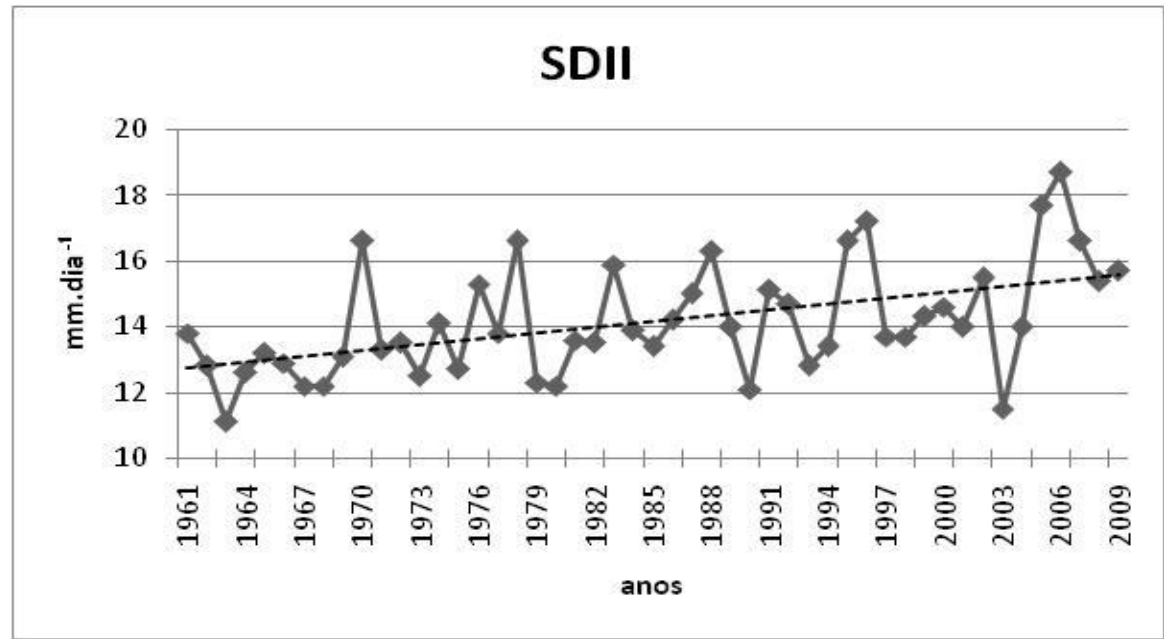

Figura 08. Densidade anual definida pela precipitação anual total dividida pelo o número de dias úmidos.

Em todos os índices que apresentaram uma tendência de aumento significativo ao longo da série, percebeu-se que o ano 2003 mostrou-se dissonante do aspecto geral dessa tendência positiva, com valores sempre abaixo dos registrados nos últimos anos da série. Isso demonstra de forma intuitiva, que, ainda que haja uma clara alteração no comportamento climático aqui especificamente a variável chuva - em uma localização qualquer, tal alteração não impossibilita a ocorrência de anos com comportamento opostos ao padrão que se detecta, pois existem variados aspectos (oceânicos, geológicos, 
atmosféricos, etc.) que interferem no clima em diversas escalas de tempo (mensais, sazonais etc.). Sendo, portanto, um aspecto natural da variabilidade do clima.

\section{CONCLUSÕES}

O índice PRCPTOT deixa claro que houve uma tendência de aumento estatisticamente significativo ao longo da série. Pelas análises apresentadas leva-se à conclusão de que a ocorrência de dias com precipitações mais intensas está aumentando, ao passo que o número de dias úmidos não apresentou qualquer tendência de crescimento no período avaliado na estação Meteorológica localizada na cidade de São Paulo. O crescimento das chuvas intensas com no mínimo $50 \mathrm{~mm} \cdot \mathrm{dia}^{-1}$ (R50mm) e do volume anual de chuvas acima do percentil 95 (R95p) reforçam a idéia de que o aumento quase contínuo do volume anual das chuvas durante o período 1961-2009 se deveu a esses fatores.

Pode-se concluir, também, que a Cidade de São Paulo tem sido mais suscetível a acontecimentos de tempestades, porém, tal fato não descarta a possibilidade de anos com baixa ocorrência de chuvas intensas e com um volume anual mais reduzido, como no ano de 2003, o qual apresentou um comportamento desarmônico com a série em praticamente todos os índices avaliados.

Conclui-se também, que, mesmo havendo uma tendência estatisticamente significativa de aumento nos índices climáticos, a existência de anos em desarmonia com a série ou parte da mesma esta dentro dos aspectos naturais da dinâmica e variabilidade do clima.

Importante se faz esclarecer que as inclinações positivas aqui detectadas demonstram, tão somente, que existiu uma tendência linear de crescimento no período estudado, e que não se pode afirmar por este trabalho, que o comportamento climático da variável precipitação na Cidade de São Paulo ocorreu como efeito das mudanças climáticas globais, podendo ser uma conseqüência de vários fatores, que vão desde a urbanização acentuada até a ação de fenômenos naturais de grande escala como a Oscilação Decadal do Pacífico.

\section{REFERÊNCIAS}

BARBETTA, P. Alberto. Estatística Aplicada às Ciências Sociais. 6a edição, Ed. UFSC, 2006.

BUSSAB, W. O. e MORETTIN, P. A. Estatística Básica. 5a edição, Ed. Saraiva, 2006.

DUFEK, A. S. e AMBRIZZI, T. Precipitation variability in São Paulo State, Brazil. Theoretical and Applied Climatology Volume 93, Numbers 3-4, 2008 
HARELL Jr, Frank E. Regression modeling strategies: with applications to linear models, logistic regression and survival analysis. New York. Ed.: Springer-Verlag, 2001.

MANTUA, Nathan J.; HARE, Steven R., ZHANG, Y.; WALLACE, John M. and FRANCIS, Robert C. A Pacific interdecadal climate oscillation with impacts on salmon production. Bulletin of the American Meteorological Society 78: 1069-1079, 1997.

NUNES, Luciana Neves, KLUCK, M. M., FACHEL, J. M. G.. Uso de imputação múltipla de dados faltantes: uma simulação utilizando dados epidemiológicos Periódico: Cad. Saúde Pública, Rio de Janeiro, 2009.

SANTOS, C. A. C.; BRITO, J. I. B. Análise dos índices de extremos para o semi-árido do Brasil e suas relações com TSM e IVDN. Revista Brasileira de Meteorologia, v. 22, n. 3, p. 303-312, 2007.

SANTOS, C. A. C. dos, BRITO, J. I. B. de, RAO, T. V. R. e MENEZES, H. E. A. Tendências dos Índices de Precipitação no Estado do Ceará. Revista Brasileira de Meteorologia, v. 24, n.1, 39-47, 2009.

SIQUEIRA, Anderlan H. B., SOUZA, L. O. , VAZ, J. C. M., MOLION, L. C. B., BERNARDO, S. de O., SALVADOR, M. de A., OLIVEIRA, J. P. P. de. Impactos do ENOS no norte e nordeste brasileiros e sua relação com a Oscilação Decadal do Pacífico. Congresso Brasileiro de Meteorologia, Florianópolis, 2006.

ZHANG, X., e YANG, F. RClindex (1.0) User Guide. Climate Research Branch Enviroment Canada, 22p, 2004. 
